\title{
Sestrin2 reduces cancer stemness via Wnt/ $\beta$-catenin signaling in colorectal cancer
}

\author{
Jinlai Wei ${ }^{1 \dagger}$, Xiangru Zheng ${ }^{2 \dagger}$, Wenjun $\mathrm{Li}^{2}$, Xiaoli $\mathrm{Li}^{3}$ and Zhongxue $\mathrm{Fu}^{1 *}{ }^{*}$
}

\begin{abstract}
Background: Colorectal cancer (CRC) is one of the most commonly diagnosed cancers in both men and women in China. In previous studies, Sestrin2 was demonstrated to have functions in CRC. However, the relationship between Sestrin2 and cancer stemness has not been reported.

Methods and results: To investigate the contribution of Sestrin2 in CRC, we performed bioinformatics analysis of The Cancer Genome Atlas datasets and found that Sestrin2 was downregulated in CRC. Using a lentivirus vector, we verified that Sestrin2 suppressed CRC cell proliferation, migration, and colony formation. Furthermore, sphere formation, flow cytometry, quantitative PCR, and western blot analysis verified the influence of Sestrin2 on cancer stemness, including the expression of cluster of differentiation 44, octamer-binding transcription factor 4 , sex-determining region Y-Box 2, CXC chemokine receptor 4, and the Wnt pathway downstream factors $\beta$-catenin and c-Myc. Consistently, the Wnt pathway activator BML-284 partially rescued the effects of Sestrin2 on the expression of proteins related to cancer stemness. Furthermore, in a mouse xenoplant model, tumors expressing Sestrin 2 were significantly reduced in size with corresponding changes in cancer stemness.
\end{abstract}

Conclusions: Collectively, our results suggest that Sestrin2 inhibits CRC cell progression by downregulating the Wnt signaling pathway. Thus, Sestrin2 may be a promising therapeutic target for CRC.

Keywords: Sestrin2, Colorectal cancer, Wnt/ $\beta$-catenin, Cancer stemness, Therapeutic target

\section{Background}

Colorectal cancer (CRC) is one of the most commonly diagnosed cancers in both men and women in China [1]. Standard anticancer therapies for CRC include surgery and chemotherapy, but the survival rate is still unsatisfactory. The failure of standard anticancer treatments might be due to cancer stem cells (CSCs) [2]. CSCs are tumor cells that have stemness properties, namely, selfrenewal, tumor initiation capacity, and long-term repopulation potential [3]. In addition to CSCs, some cancer cells also have stemness properties, and these cells might

\footnotetext{
*Correspondence: fzx@hospital.cqmu.edu.cn

${ }^{\dagger}$ Jinlai Wei and Xiangru Zheng contributed to the work equally

1 Department of Gastrointestinal Surgery, The First Affiliated Hospital of Chongqing Medical University, Chongqing 400016, China

Full list of author information is available at the end of the article
}

be a result of the transformation of typical stem cells in the tissue or emerge from nonstem cell regulation due to exposure to stemness factors [4]. Targeting CSCs would be a promising therapeutic method. Therefore, investigating CSCs to find new molecules and mechanisms in CRC would reveal novel therapeutic targets.

Sestrin family members, including Sestrin1, Sestrin2, and Sestrin3, can be induced by cellular stresses, such as ER stress, DNA damage and ROS accumulation [5-7]. In the Sestrin family, Sestrin2 has shown regulatory activities in some cancers [8]. Knocking down Sestrin2 in non-small cell lung cancer cells suppressed lung cancer progression [9]. Downregulation of Sestrin2 promoted cell proliferation, migration, and ROS production in endometrial cancer cells [10]. In CRC, our previous study demonstrated reduced expression of Sestrin2 relative to normal colorectal epithelial tissues [11]. Other

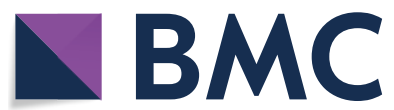

(c) The Author(s) 2022. Open Access This article is licensed under a Creative Commons Attribution 4.0 International License, which permits use, sharing, adaptation, distribution and reproduction in any medium or format, as long as you give appropriate credit to the original author(s) and the source, provide a link to the Creative Commons licence, and indicate if changes were made. The images or other third party material in this article are included in the article's Creative Commons licence, unless indicated otherwise in a credit line to the material. If material is not included in the article's Creative Commons licence and your intended use is not permitted by statutory regulation or exceeds the permitted use, you will need to obtain permission directly from the copyright holder. To view a copy of this licence, visit http://creativecommons.org/licenses/by/4.0/. The Creative Commons Public Domain Dedication waiver (http://creativeco mmons.org/publicdomain/zero/1.0/) applies to the data made available in this article, unless otherwise stated in a credit line to the data. 
studies have shown that Sestrin2 has many other functions in CRC; for example, Sestrin2 is involved in inducing the apoptotic process of HCT116 CRC cells [12], and its overexpression inhibits the migration, invasion, and growth of CRC [13]. However, the relationship between Sestrin2 and CRC stemness has not been previously reported.

This study aimed to investigate the role of sestrin 2 in the cancer stemness of CRC. First, the effects of upregulating Sestrin 2 on the survival, migration, and colony formation of CRC cells were studied, and the expression of cancer stemness in CRC cells with high Sestrin2 expression was detected. Second, the relationship between Sestrin2 and Wnt signaling was explored. Finally, we observed that upregulation of Sestrin2 inhibited tumor growth in CRC cells in vivo. Our results suggest that Sestrin2 inhibits CRC cell progression by downregulating the Wnt signaling pathway.

\section{Materials and methods Cell culture}

The human CRC cell lines were purchased from Shanghai Zhong Qiao Xin Zhou Biotechnology Co., Ltd. (Shanghai, China). HCT-116 cells were cultured in McCoy's 5A medium (Biological Industries, Beit HaEmek, Israel), and SW620 cells were cultured in RPMI 1640 medium (Biological Industries) supplemented with 10\% fetal bovine serum (FBS) (Biological Industries). Cells were cultured at $37{ }^{\circ} \mathrm{C}$ with $5 \% \mathrm{CO}_{2}$ in humidified incubators.

\section{Infection with lentiviral constructs}

Lentiviral constructs expressing Sestrin2 (LV-Sestrin2) and a nontargeted green fluorescence protein (GFP) virus (LV-GFP) were purchased from GeneChem (Shanghai, China). Cells $\left(1.5 \times 10^{4}\right)$ were plated in 24-well plates on the day before transfection. Lentiviral constructs were transduced at a multiplicity of infection of 10 in HCT116 and SW620 cells using HiTransG P transfection reagent (GeneChem). The infected cells were maintained in fresh medium without FBS for $12 \mathrm{~h}$ and then washed with phosphate-buffered saline (PBS), after which the medium was replaced with fresh medium supplemented with $10 \%$ FBS. Puromycin $(2 \mu \mathrm{g} / \mathrm{mL}$; Beyotime, Beijing, China) was used to generate stable expression cell lines in HCT116 and SW620 cells.

\section{Quantitative PCR}

The RNAsimple Total RNA Kit (TIANGEN, Beijing, China) was used to extract total RNA according to the manufacturer's instructions. All-in-One cDNA Synthesis SuperMix (Bimake, USA) was used to generate firststrand cDNA. The expression level of the cDNAs was normalized to that of $\beta$-actin by the comparative $\mathrm{CT}$ method. The primer sequences used in this study are provided in Supplementary Table 1.

\section{Cell proliferation assay}

A total of 500 cells per well were plated in the wells of a 96-well plate. Cell viability was measured using Cell Counting Kit-8 (CCK-8) (Bimake) and the Thermo Scientific $^{\mathrm{TM}}$ Varioskan $^{\mathrm{TM}}$ LUX Multimode microplate reader (Thermo Fisher Scientific, Waltham, MA, USA) at $450 \mathrm{~nm}$ once a day for 7 days.

\section{Extreme limiting dilution assay in vitro}

The procedure of the extreme limiting dilution assay (ELDA) was the same as that described previously [14]. Briefly, cells were seeded in U-bottom 96-well plates at final cell concentrations of 1000,100,10, and 1 per well with $200 \mu \mathrm{L}$ SCM. After 2 weeks, the wells containing cell spheres were counted, and empty wells were excluded. The frequency of CSCs was calculated on the website (http://bioinf.wehi.edu.au/software/elda/) [15].

\section{Sphere formation}

Sphere cells were induced from adherent cells for 2 weeks in stem cell medium (SCM), which is a DMEM/F-12 medium supplemented with epidermal growth factor (20 ng/mL, MedChemExpress, MCE), basic fibroblast growth factor $(20 \mathrm{ng} / \mathrm{mL}, \mathrm{MCE})$, and B-27 (2\%; Invitrogen, Carlsbad, CA, USA). A total of 200 sphere cells were plated into each 96-well plate with $200 \mu \mathrm{L}$ SCM. The number of spheres was counted after 2 weeks. For the second sphere assay, sphere cells were separated using ACCUTASE (YEASEN). A total of 200 cells were plated per well in each 96-well plate with $200 \mu \mathrm{L}$ SCM. The number of spheres was counted after 2 weeks.

\section{Soft agar colony formation assay}

To evaluate the anchorage-independent growth of LVSestrin2 cell lines, a concentration of $1 \times 10^{4}$ cells was suspended in SCM containing $0.36 \%$ agar and poured on an agar bed (SCM with $0.75 \%$ agar). After 3 weeks, the sphere number was counted after $0.04 \%$ crystal violet staining.

\section{Wound healing assay}

HCT116 cells and SW620 cells $\left(4 \times 10^{5}\right.$ cell/well $)$ were seeded into 6-well plates. On the next day, straight lines were scratched on the cell monolayer using a $200 \mu \mathrm{L}$ pipette tip. After washing away the cell debris with PBS, fresh culture medium with $2 \%$ FBS was added. Images of the migratory cells were captured at $0 \mathrm{~h}, 24 \mathrm{~h}$ and $48 \mathrm{~h}$. The relative cell migration rate of each group (initial area 
minus $48 \mathrm{~h}$ scratch area) was normalized against the scratched area at $0 \mathrm{~h}$ (ImageJ software 1.52a).

\section{Transwell assay}

A total of $1 \times 10^{6}$ cells were added to the upper chamber (0.8 $\mu \mathrm{M}$ in 6-well plates) of a Transwell chamber (JET, China) in serum-free culture medium. The lower chamber contained medium supplemented with 10\% FBS. After $24 \mathrm{~h}$ of culture, the cells on the upper side of the filter membrane were removed. The rest of the cells were fixed with 5\% paraformaldehyde for $20 \mathrm{~min}$, dyed using Crystal Violet Staining Solution (Beyotime, China), photographed at $200 \times$ and counted in five random fields.

\section{Flow cytometry}

Cells were detached into single cells, washed with cold PBS, preincubated with Human TruStain $\mathrm{FCX}^{\mathrm{TM}}(422,301$; BioLegend, San Diego, CA, USA), and then incubated with CD44-APC (B265921; BioLegend) on ice in the dark. The cells were assayed by flow cytometry.

\section{Wnt/ $\beta$-catenin pathway inhibition assay}

HCT-116 cells in the LV-Sestrin2 and LV-GFP groups were treated with $0.5 \mu \mathrm{M}$ BML-284 (Wnt signaling activator; MedChemExpress, Monmouth Junction, NJ, USA) in dimethyl sulfoxide for $24 \mathrm{~h}$ and then collected for western blot analysis. In the sphere formation assay, $0.1 \mu \mathrm{M}$ BML-284 was mixed with the cells and then they were plated in 96-well plates with SCM. The number of spheres was counted after 2 weeks.

\section{Western blot analysis}

Cells were washed with PBS and lysed in RIPA buffer with Protease Inhibitor Cocktail (Bimake) on ice. Cell lysates were centrifuged $(12,000 \mathrm{rpm})$ at $4{ }^{\circ} \mathrm{C}$ for $20 \mathrm{~min}$ and then quantified using the BCA Protein Assay Kit (Beyotime). The lysate was denatured with sodium dodecyl sulfate-polyacrylamide gel electrophoresis (SDSPAGE) sample loading buffer (Beyotime), followed by SDS-PAGE and electrotransfer to polyvinylidene difluoride membranes (Millipore, Darmstadt, Germany). The membranes were incubated overnight at $4{ }^{\circ} \mathrm{C}$ with anti-Sestrin2 (Proteintech Group 10795-1-AP 1:1000), anti-CD44 (Proteintech Group 15675-1-1AP 1:1000), anti-Sox2 (Proteintech Group 11064-1-AP 1:1000), antiOct4 (Proteintech Group 11263-1-AP 1:1000), antiCXC chemokine receptor 4 (Cxcr4) (Proteintech Group 11073-2-AP 1:1000), anti- $\beta$-catenin (Proteintech Group 51067-2-AP 1:1000) and anti- $\beta$-actin (Proteintech Group 20536-1-AP 1:1000) at the appropriate dilution and then incubated with the secondary antibody at room temperature for $2 \mathrm{~h}$. The bands were visualized using BeyoECL Plus (Beyotime).

\section{Xenograft mouse model}

A xenograft mouse model was established by subcutaneous injection. One million HCT-116 cells in the LVSestrin2 and LV-GFP groups were injected into the hips of 6-week-old female BALB/c-nu mice (Beijing Vitalstar Biotechnology, China). All animal studies were approved by the Ethics Committee of Chongqing Medical University. The tumor size was measured 5 days later and then every 3 days. The tumor volume $\left(\mathrm{V}=\mathrm{l} \times \mathrm{w}^{2} / 2\right)$ was calculated by measuring the length (l) and width (w). Mice were euthanized in a $\mathrm{CO}_{2}$ chamber 20 days after cell injection.

\section{Immunohistochemistry (IHC)}

IHC assays were conducted as reported previously [16]. Antibody against Sestrin2 (Proteintech Group 10795-1AP 1:500), Ki-67 (Proteintech Group, 27309-1-AP, 1:500) or $\beta$-catenin (Proteintech Group 51067-2-AP 1:500) were incubated at room temperature for $2 \mathrm{~h}$. The biotinylated goat anti-rabbit antibody (Beyotime China, A0279, 1:200) was used as the secondary antibody. The diaminobenzidine tetrachloride (Beyotime China, P0203) was used for staining at room temperature for $1 \mathrm{~min}$.

\section{Bioinformatics analysis}

The colorectal cancer dataset comprised mRNA-seq data from TCGA tumors (https://tcga-data.nci.nih.gov/tcga/). The two-gene correlation map was generated by the $\mathrm{R}$ software package ggstatsplot, and the multigene correlation map was displayed by the R software package heatmap. We used Spearman's correlation analysis to describe the correlation between the quantitative variables without a normal distribution. A $P$ value of less than 0.05 was considered statistically significant.

\section{Statistical analysis}

Except where otherwise noted, the experiments were repeated at least three times. All statistical analyses were performed using GraphPad Prism Version 8. The MannWhitney test or $\mathrm{T}$ test was used to evaluate the significance of the differences between two groups of data. $P \leq 0.05$ was considered statistically significant. Medians were used to summarize the data.

\section{Results}

Sestrin2 inhibits CRC cell proliferation, migration, and colony formation

To evaluate the effects of Sestrin 2 on CRC, we first investigated the mRNA expression of Sestrin2 compared to the 
corresponding adjacent tissues in The Cancer Genome Atlas datasets using the UALCAN website (UALCAN: http://ualcan.path.uab.edu) (Fig. 1A) [17]. The results showed that Sestrin2 was significantly downregulated in both colon adenocarcinoma (COAD) and rectum adenocarcinoma (READ).

To investigate the effects of Sestrin2 on cancer phenotypes in vitro, we introduced LV-Sestrin2 and LVGFP lentivirus particles into HCT116 and SW620 cells, and proliferation, migration, and colony formation were evaluated. To detect the effect of Sestrin 2 on CRC proliferation, a CCK- 8 assay was performed, and the results showed that the absorbance of the LV-Sestrin2 group began to differ significantly from that of the LV-GFP group after 3 days and 4 days in the HCT-116 and SW620 cells, respectively (Fig. 1B). These results suggested that Sestrin2 inhibited the proliferation of CRC cells.

In addition, the colony formation assay showed that the number of colonies formed was decreased from $164.0 \pm 10.44$ in LV-GFP to $97.67 \pm 11.59$ in LV-Sestrin2 in HCT-116 cells and from $122.0 \pm 6.245$ to $100.7 \pm 4.619$ in SW620 cells (Fig. 1C).

Furthermore, to investigate the migration ability, Transwell and wound healing assays were conducted. The Transwell assay results showed that the migration of LVSestrin2-transduced CRC cells was inhibited (Fig. 1D). The wound healing assay also showed that HCT116 and SW620 cells healed less than $60 \%$ and more than $30 \%$ of the area, respectively, in the LV-GFP group; however, the healing ability was reduced to less than $20 \%$ and nearly $10 \%$ after LV-Sestein2 transduction in these two cell lines (Fig. 1E). A similar trend was also observed in the invasion assay (Additional file 1: Figure S1). The data suggested that Sestrin2 inhibited migration and invasion in CRC. These results demonstrate that Sestrin2 is downregulated in CRC patients and has antiproliferative and antimigratory effects on CRC cells.

\section{Sestrin2 inhibits the cancer stemness in CRC cells}

CSCs play a significant role in cancer progression, such as tumor growth, recurrence, and metastasis [18]. Therefore, we hypothesized that Sestrin 2 affects CSCs in CRC cells. Self-renewal and tumor initiation are basic characteristics of CSCs [19]. To identify the effect of Sestrin2 on self-renewal of CRC adhering cells, a sphere assay was used. The sphere assay results showed that in HCT116 cells, the LV-GFP cells formed $32.33 \pm 6.03$ colonies, while LV-Sestrin2 formed $14.67 \pm 4.51$ colonies. The SW620 cell line had $41.33 \pm 8.02$ colonies in the LV-GFP group and $11.67 \pm 4.16$ colonies in the LV-Sestrin2 group (Fig. 2A). To investigate the effect of Sestrin2 on the selfrenewal ability of CSCs, a second sphere formation assay was performed [20]. Unsurprisingly, the colony number formed in the second sphere formation assay had a similar trend to that of the first sphere formation assay. LVGFP HCT-116 sphere cells formed $7.33 \pm 2.31$ colonies, LV-Sestrin2 formed $2.33 \pm 0.58$ colonies, and SW620 cells formed $8.67 \pm 0.58$ and $1.33 \pm 1.53$ colonies, respectively, suggesting that Sestrin2 inhibited CSC self-renewal in CRC (Fig. 2A).

The strength of anchorage-independent growth in soft agar assays reflects the tumorigenesis of the cells [21]. Soft agar assays are able to imitate an in vivo microenvironment [22]. HCT-116 and SW620 cells in the LV-GFP and LV-Sestrin2 groups were cultured in soft agar to form colonies over 3 weeks. The average colony sizes of the LV-GFP and LV-Sestrin2 HCT-116 colonies were $5816.67 \pm 1190.17$ and $2035.67 \pm 941.48$, respectively, while the average sizes of the SW620 cells were $2067.67 \pm 739.55$ and $697.33 \pm 386.35$ pixels, respectively (Fig. 2A). Moreover, to determine the number of colorectal cancer-initiating cells, we tested colony formation using limited dilutions of cells, and the results showed that LV-Sestrin2 reduced the CSCs in the HCT-116 and SW620 cell lines (Table 1). These data suggest that Sestrin2 decreased self-renewal and tumor formation ability in CRC.

In addition, molecules related to cancer stemness were evaluated. Cell surface proteins are used as CSC markers for different types of tumors, for example, cluster of differentiation 34-positive (CD34+)/CD38 - for leukemia cells, CD13/CD45/CD90 for liver cancer, CD117/ CD90/epithelial cell adhesion molecule for lung cancer [23, 24], and CD44 for colon and gastric cancers [25]. We used flow cytometry to investigate the expression strength of the stemness biomarker CD44 and the number of CD44+ cells. Flow cytometry showed that both the percentage of CD44+ cells and the expression strength of

\footnotetext{
(See figure on next page.)

Fig. 1 Sestrin2 has anticancer effects on CRC cells. A Sestrin2 was downregulated in both COAD $\left(P=0.28 \times 10^{-14}\right)$ and READ $(P=0.024)$ compared to normal tissue in TCGA. B Cell proliferation was markedly inhibited after Sestrin2 upregulation, as determined by the CCK-8 assay. $\left({ }^{*} P=0.05\right.$; Mann-Whitney test; connected by medians) C The colony formation assay showed that the number of colonies formed by LV-Sestrin2-infected cells was smaller than that of LV-GFP-infected cells. The right panel shows the number of colonies ${ }^{*} P=0.05$; Mann-Whitney test; lines showed medians). D Cell migration was detected by Transwell assays. The cell number was counted by ImageJ and normalized to the LV-GFP group (right panel) ${ }^{*} P=0.05 ;$ Mann-Whitney test; lines showed medians). E Cell migration was measured by the wound healing assay. The area of migration was quantified as the mean \pm standard deviation (SD) and normalized to the respective LV-GFP group (right panel); ${ }^{*} P=0.05$; Mann-Whitney test; lines showed medians
} 
A

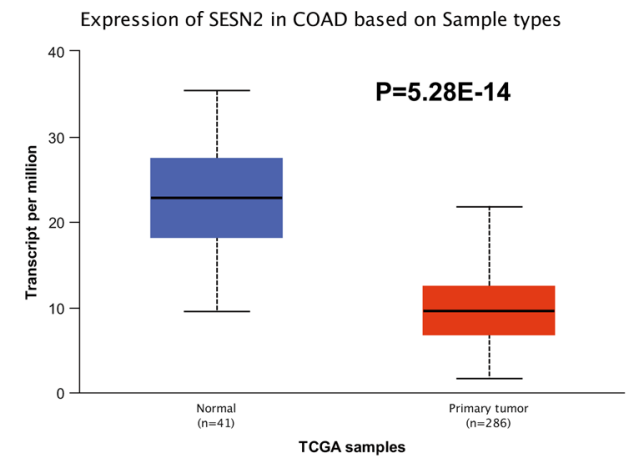

B

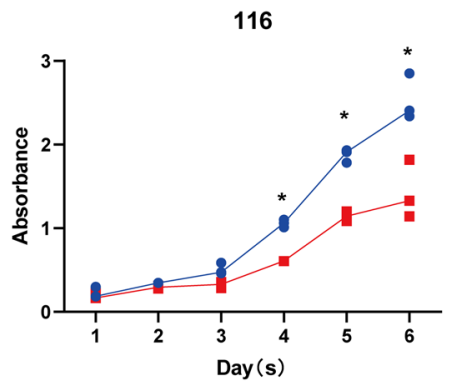

- 116-M

- 116-SU

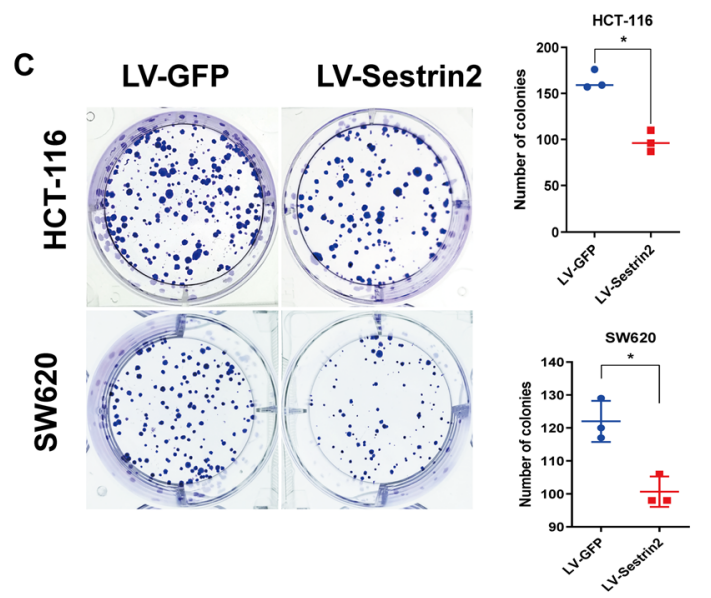

E

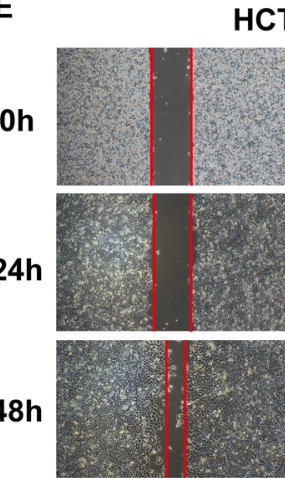

LV-GFP
HCT-116

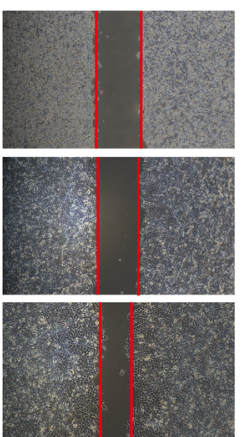

LV-Sestrin2

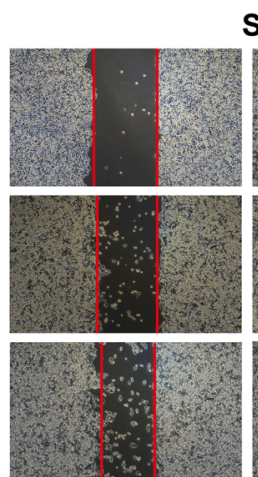

LV-GFP
- 29-M
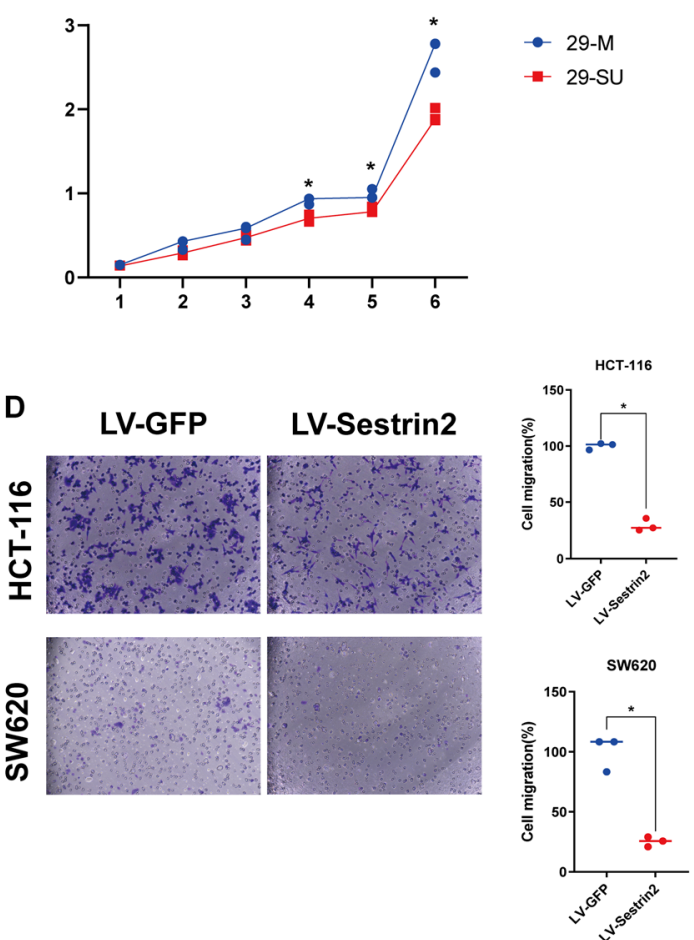

SW620

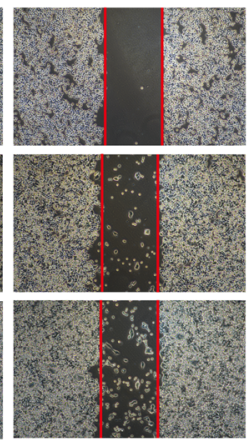

LV-Sestrin2
F
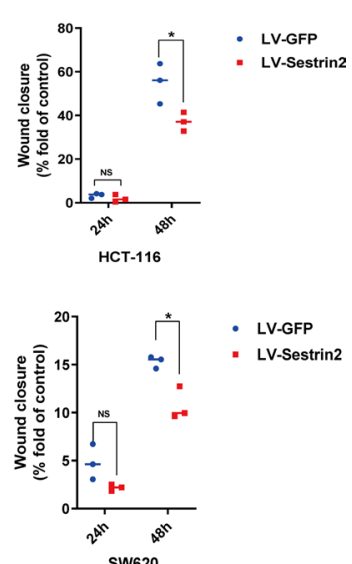

Fig. 1 (See legend on previous page.) 
CD44 were decreased in Sestrin2-transfected CRC cells (Fig. 2B).

The stemness state is governed by stem cell factors such as the four Yamanaka factors sex-determining region Y-Box 2 (Sox2), octamer-binding transcription factor 4 (Oct4), Kruppel-like factor 4, and c-Myc [26]. Stemnessrelated markers, including CD44, Oct4, Sox2, and Cxcr4, were investigated by western blotting and qPRC to illustrate the effect of Sestrin2 on protein expression and mRNA transcription. The results showed that LV-Sestrin2 downregulated stemness-related markers at both the mRNA and protein levels in HCT-116 and SW620 cells (Fig. 2C-D, Additional file 2: Figure S2A).

Furthermore, whether Sestrin2 affected stemness in CSCs was investigated by western blot. Sphere cells were induced from adherent cells in SCM for 2 weeks, and then western blotting was conducted. The results showed similar protein expression trends in sphere cells, which hinted that Sestrin2 was able to reduce stemness in CSCs (Additional file 2: Figure S2B).

Epithelial-mesenchymal transition (EMT) promotes cancer stemness. We also investigated the expression of E-cadherin, an EMT negative correlation marker, in LV-GFP and LV-Sestrin2 cells by western blotting (Additional file 2: Figure S2C). The results showed that Sestrin2 increased the expression of E-cadherin, which indicated that Sesterin2 negatively regulated EMT and cancer stemness.

These data prove that Sestrin2 suppresses the expression of stemness-related markers and reduces cancer stemness in CRC cells.

\section{Sestrin2 reduces cancer stemness via the $W n t / \beta$-catenin pathway}

Wnt signaling is one of the key pathways that regulate development and stemness in cancer [27]. The function of the Wnt pathway in CSCs is commonly accepted and it depends on the amount of $\beta$-Catenin in the cytoplasm $[28,29]$. $\beta$-catenin is able to activate not only proliferation and transmission factors, such as c-Myc, c-Jun, and CCND1 (the gene encoding cyclin D1) but also epidermal growth factor receptors, such as CD44 and CD133. Activation of the Wnt/ $\beta$-Catenin pathway increases the number of CSCs by regulating self-renewal and homeostasis in cancer cells [30]. We observed the protein expression of $\beta$-catenin and the downstream target c-Myc. Our observations showed that the expression of $\beta$-catenin and c-Myc was downregulated (Fig. 3A). Furthermore, we used the $\mathrm{Wnt} / \beta$-catenin pathway activator BML-284 to rescue the effects of Sestrin2 and found that BML-284 was able to partly rescue the effects of Sestrin2 using the sphere assay (Fig. 3B-C), consistent with the western blot analyses in HCT-116 cells (Fig. 3D-E). Our findings suggest that Sestrin2 regulates cancer stemness via the Wnt/ $\beta$-catenin pathway.

In addition, reactive oxygen species (ROS) were reported to play an important role in maintaining cancer stemness [31]. Sestrin2 was able to suppress ROS in cancer cells [32]. To investigate whether sestrin 2 affects cancer stemness by regulating ROS, scavengers of ROS, sodium pyruvate and carboxy-PTIO were used. The results showed that scavengers of ROS, although they slightly downregulated the protein expression of CD44, were unable to rescue the CD44 inhibition caused by Sestrin2 overexpression, regardless of whether the scavenger was used alone or in combination (Additional file 3: Figure S3). These data indicate that Sestrin2 does not regulate cancer stemness through ROS.

\section{Sestrin2 inhibits cancer stemness in vivo}

To evaluate the effects of Sestrin2 in vivo, a subcutaneous xenotransplant tumor model was used. A concentration of $1 \times 10^{7}$ cells from the LV-Sestrin 2 or LV-GFP groups was injected into each of five female $\mathrm{BALB} / \mathrm{c}-\mathrm{nu}$ mice. The xenotransplant tumors were observed 5 days later and then every 3 days. Twenty days after cell injection, the mice were euthanized, and the tumors were removed (Fig. 4A-B). The growth rate of the tumors produced by HCT116-Sestrin2 cells was significantly slower than that of tumors produced by HCT116-GFP cells, although the weights of the mice were not significantly different (Fig. 4C, Additional file 4: Figure S4A). After the tumors were removed, the volume and weight were measured again, and the tumors from the LV-Sestrin2 group were inhibited $(P=0.0101$ in volume, $P=0.0334$ in weight, Fig. 4D-E).

\footnotetext{
(See figure on next page.)

Fig. 2 Sestrin2 inhibits cancer stemness in CRC cells. A The cell sphere formation assay (top), second cell sphere formation (middle) and soft agar colony formation assay (bottom) showed that the number of spheres and the average area of the soft agar colonies were both decreased; ${ }^{*} P=0.05$; Mann-Whitney test; lines showed medians. B Flow cytometry was used to detect the percentages of CD44+ cells. The percentages of CD44+ cells in the LV-GFP group were larger than those in the LV-Sestrin2 group ( ${ }^{*} P=0.05$; Mann-Whitney test; lines showed medians). $C$ Representative western blot images of the effect of LV-Sestrin2 on the expression levels of Sestrin2, Sox2, Oct4, Cxcr4, and CD44. $\beta$-actin was used as a loading control. D The relative mRNA expression of Sestrin2, Sox2, Oct4, CxCr4, and CD44 in LV-Sestrin2 CRC cells normalized to the LV-GFP group ${ }^{*} P<0.05$, ${ }^{*} P<0.01,{ }^{* * *} P<0.001, T$ test $)$
} 
A
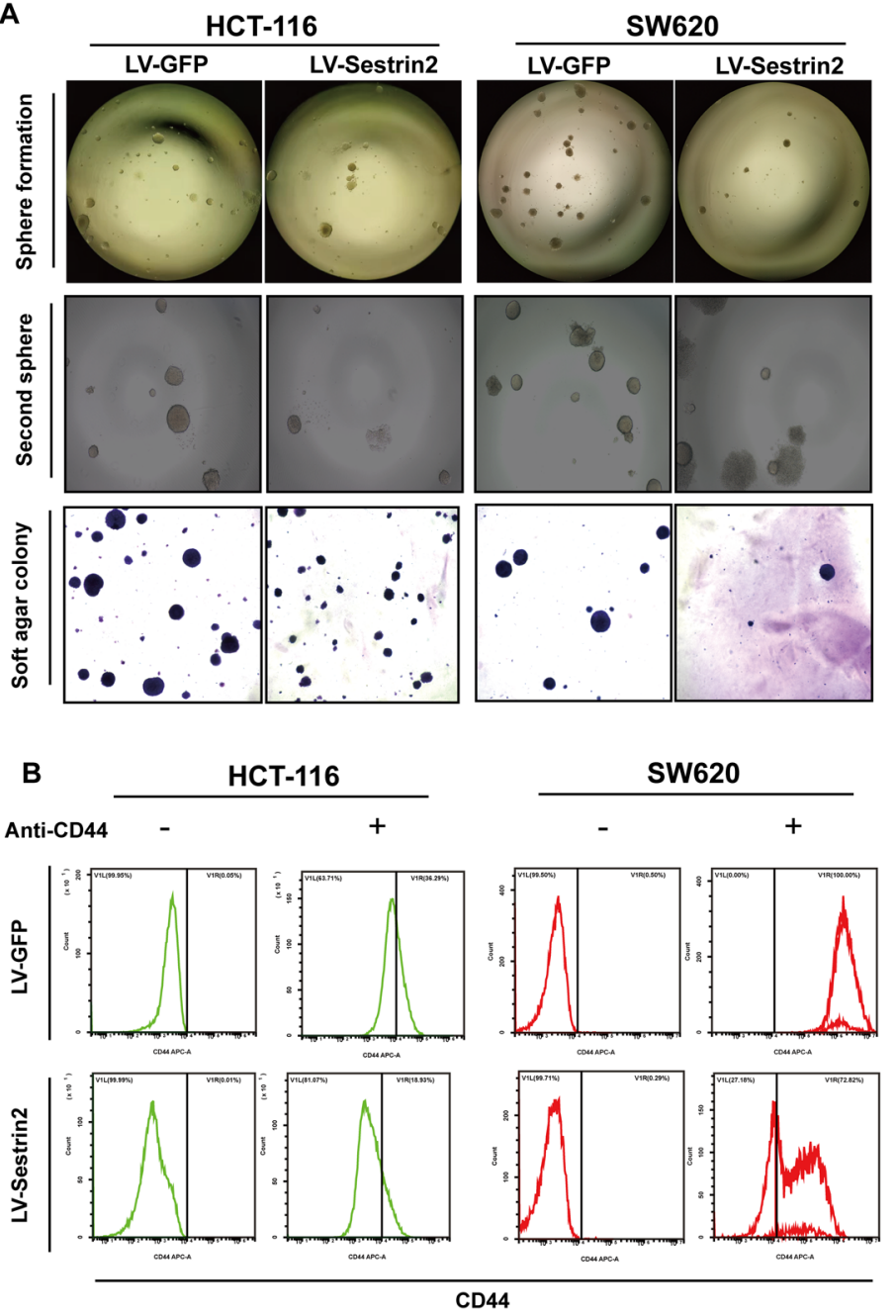

D

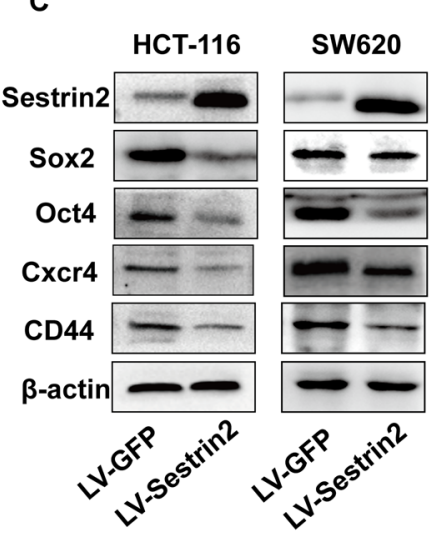

SW620
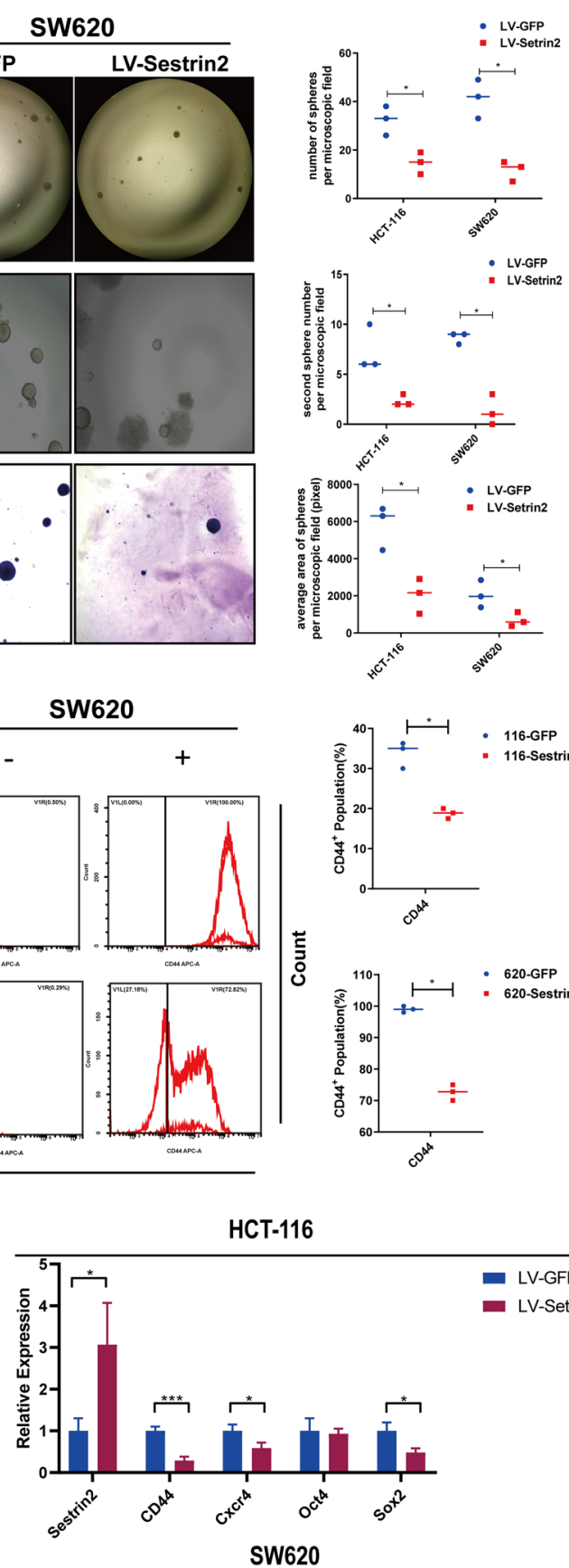

కั่
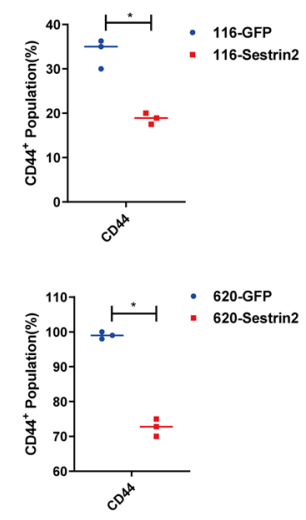

IV-GFP

- LV-Setrin2

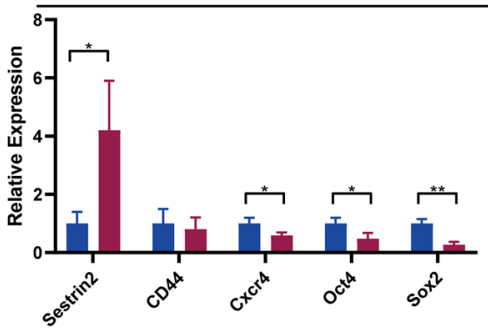

Fig. 2 (See legend on previous page.) 


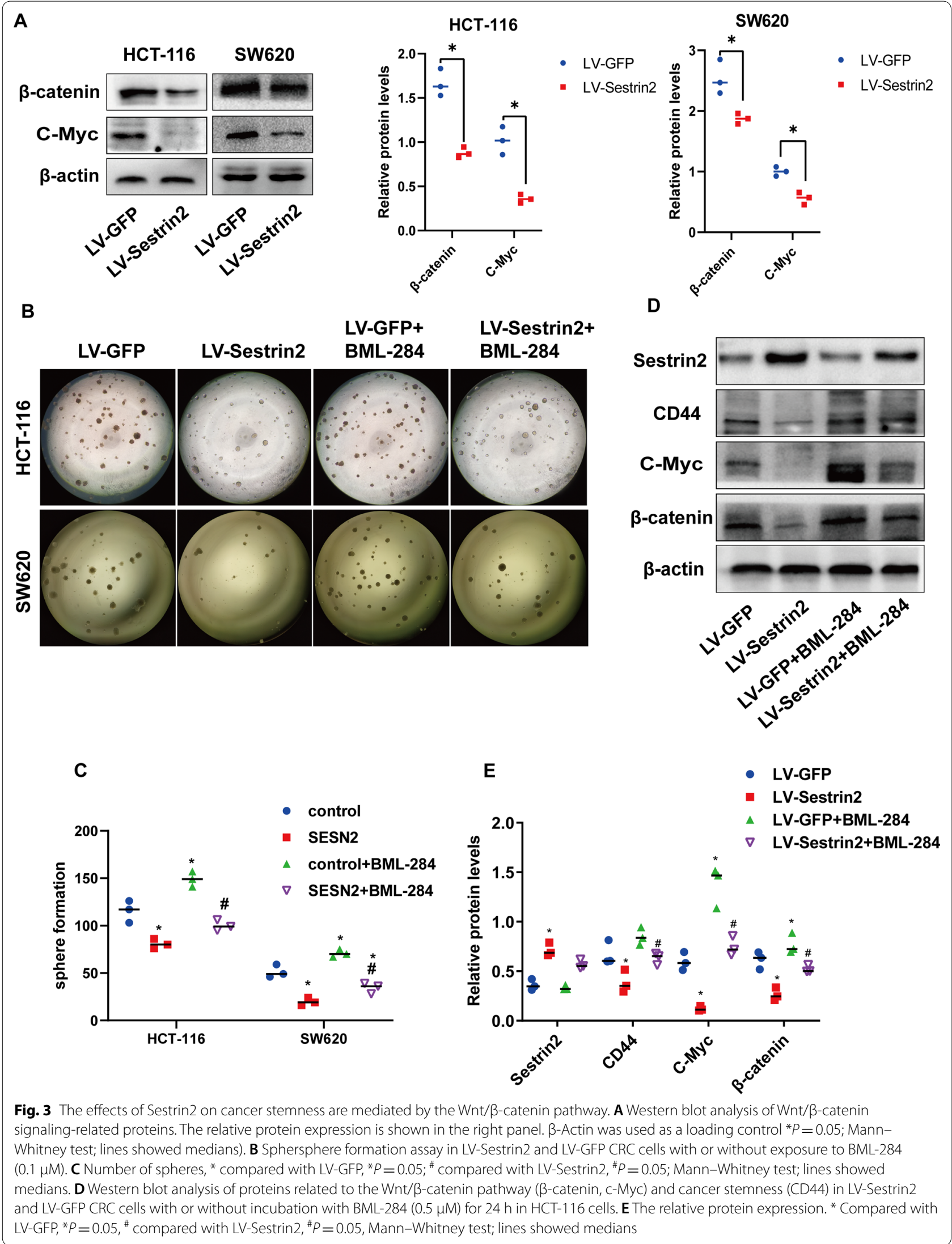


Table 1 Limiting dilution data showing the effect of Sestrin2 expression on the frequency of CSCS

\begin{tabular}{|c|c|c|c|c|c|c|c|}
\hline Cells seeded & & 1 & 10 & 100 & 1000 & $\begin{array}{l}\text { Estimated frequency } \\
\text { of CSCs }\end{array}$ & $P$ value \\
\hline \multirow[t]{2}{*}{ HCT-116 } & LV-GFP & $62 / 78$ & $152 / 162$ & $89 / 92$ & $24 / 24$ & 1 in 6 cells & 0.087 \\
\hline & LV-Sstrin2 & $78 / 105$ & $136 / 170$ & $86 / 91$ & $24 / 24$ & 1 in 7 cells & \\
\hline \multirow[t]{2}{*}{ SW620 } & LV-GFP & $45 / 90$ & $101 / 145$ & $76 / 94$ & $24 / 24$ & 1 in 19 cells & $4.24 \times 10-8$ \\
\hline & LV-Sstrin2 & $51 / 128$ & $96 / 172$ & $74 / 96$ & $22 / 24$ & 1 in 35 cells & \\
\hline
\end{tabular}

We then performed IHC of the cell proliferation markers Ki-67 and $\beta$-catenin in the tumor tissues to determine the effect of LV-Sestrin2 in vivo. As expected, the expression of Ki-67 and $\beta$-catenin in the LV-Sestrin2 group was lower than that in the LV-GFP group (Fig. 4F). Furthermore, we observed the CSCrelated protein expression of xenotransplant tumors by western blot analysis. The results showed that upregulation of Sestrin2 inhibited the protein levels of Sox2, c-Myc, and $\beta$-catenin in xenotransplant tumors (Fig. 4G, Additional file 4: Figure S4B). The efficiency of tumor formation and cancer stemness was decreased by Sestrin 2 in vivo.

Finally, to confirm our findings in CRC patients, the mRNA expression correlations between Sestrin2 and CD44, Oct4, Sox2, Cxcr4, c-Myc and $\beta$-catenin were detected by $\mathrm{R}$ using TCGA data. The mRNA expression of CD44, $\beta$-catenin, Cxcr4 and c-Myc was negatively related to Sestrin2, suggesting when upregulating sestrin 2 the other factors might downregulate, which was consistent with our in vitro results (Fig. 4H). The expression of Sestrin2 was negatively related to cancer stemness in TCGA data.

In summary, Sestrin2 inhibits the growth of CRC in vivo by downregulating cancer stemness.

\section{Discussion}

Previous studies have introduced the role of Sestrin2 in CRC. In this study, we further investigated the role of Sestrin2 in CRC and found Sestrin2 inhibits cancer stemness both in vivo and in vitro. Using primary and secondary sphere formation assays to detect the self-renewal of
CRC cells, we found that Sestrin 2 upregulation inhibited sphere formation of CRC cells. Consistently, ELDA suggested that Sestrin2 inhibited their sphere forming ability. Furthermore, the expression of CD44, a key marker for CRC, and Oct4, Sox2, and Cxcr4, stem cell factors, was also decreased. Overall, our data suggested that Sestrin2 not only reduces self-renewal but also inhibits initial ability of CRCs to form.

Sestrin2 may be a therapeutic target. First, CSCs play a very important role in cancer, including maintenance, self-renewal, division, and tumor development [33]. As tumor propagation initiators, CSCs are considered to be a promising therapeutic target [34]. In addition, in this study, Sestrin 2 targeting the Wnt/ $\beta$-catenin pathway reduced the cancer stemness of CRC cells, and Sestrin2 inhibited the proliferation, migration, and colony formation of CRC cells. Treatment targeting Sestrin2 would affect the major tumor biological characteristics of CRC. Thus, Sestin2 may be a promising therapeutic target for CRC.

EMT and CSCs are two closely related processes in tumor progression and therapeutic resistance [35]. In previous studies, Sestrin2 was shown to inhibit EMT [36, 37]. These results provide evidence that Sestrin 2 regulates cancer stemness.

Sestrin2 is an anticancer molecule. In CRC, the decreased expression of Sestrin2 in patients predicts unfavorable outcomes, and Sestrin2 is also an important facilitator of the p53-mediated control of cancer cell growth [11, 38]. Moreover, Sestrin2 has been shown to function as a tumor suppressor in lung cancer, hepatocellular carcinoma, and melanoma [39-41]. Antitumor

\footnotetext{
(See figure on next page.)

Fig. 4 Sestrin2 inhibits cancer stemness in vivo. A LV-Sestrin2 and LV-GFP cells were transplanted into the hips of mice and after 3 weeks B The tumors were excised. C Tumor volume data ( ${ }^{*} P=0.008,{ }^{*} P=0.02$, Mann-Whitney test; connected by medians). $\mathbf{D}$ The tumor volumes were measured after removal from the mice ${ }^{* *} P=0.008$, Mann-Whitney test; lines showed medians). E The tumor weights were measured $\left({ }^{*} P=0.02\right.$, Mann-Whitney test; lines showed medians). F HIC of Sestrin2, Ki-67 and $\beta$-catenin. G Western blots showing the protein expression of Sestrin2, CD44, Sox2, c-Myc, and $\beta$-catenin in mouse tumors. $\beta$-Actin was used as a loading control. $\mathbf{H}$ A heatmap of the correlations between CD44, $\beta$-catenin (CTNNB1), CXCR4, C-Myc (MYC), OCT4 (POU5F1), SOX2 and Sestrin2 (SESN2). Red represents a positive correlation, blue represents a negative correlation, and darker colors represent stronger correlations. Asterisks represent levels of significance $\left({ }^{*} P<0.05,{ }^{* *} P<0.01, T\right.$ test)
} 
A

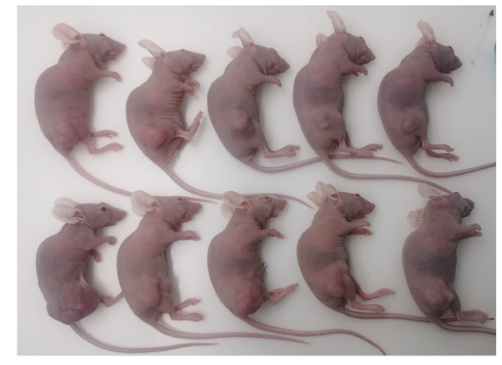

C

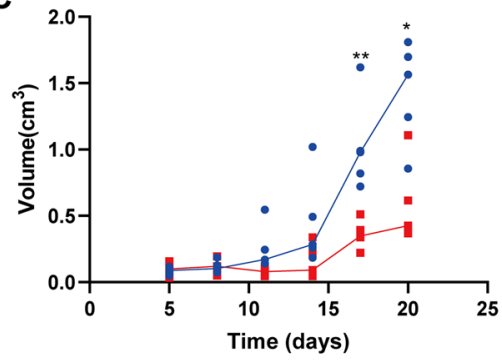

$\mathbf{F}$
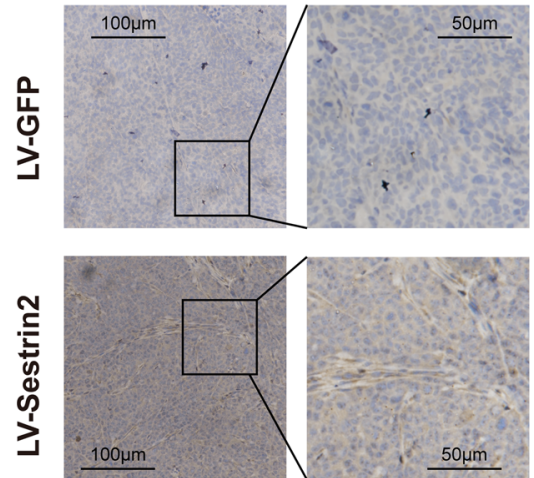

Sestrin2

G

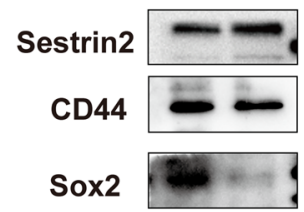

C-Myc

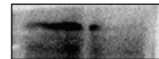

$\beta$-catenin

$\beta$-Actin

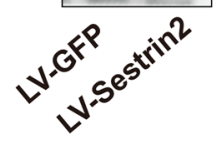

B

LV-Sestrin2

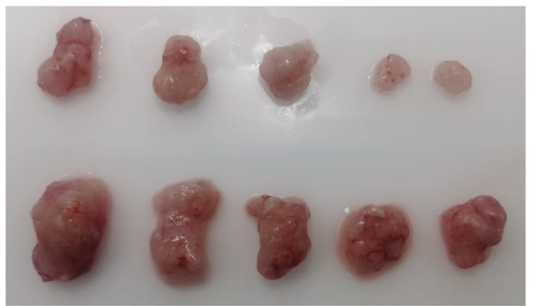

LV-Sestrin2

D

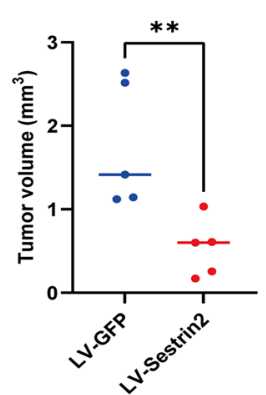

E

$\rightarrow$ LV-GFP
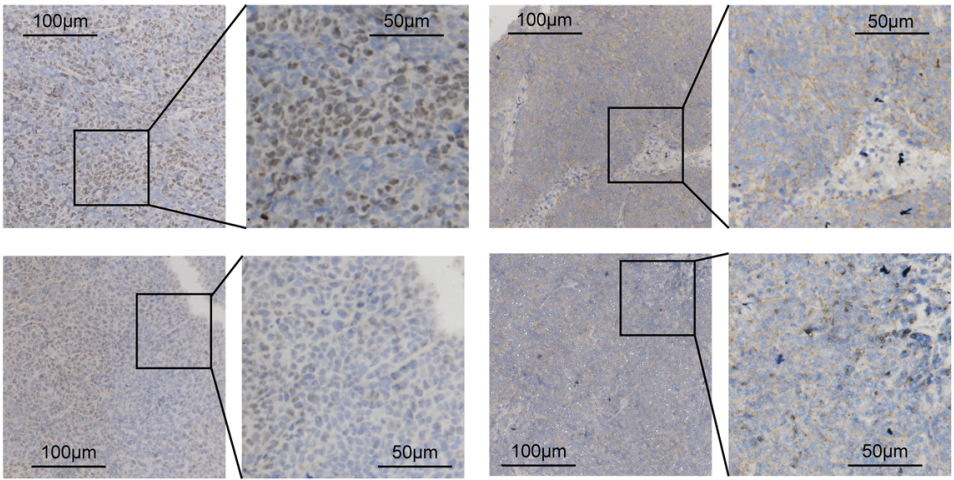

Ki-67

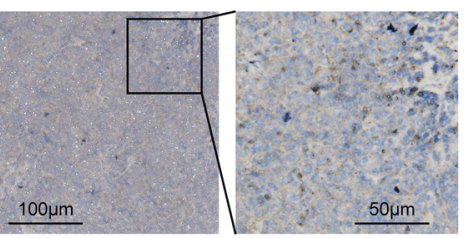

$\beta$-catenin

H

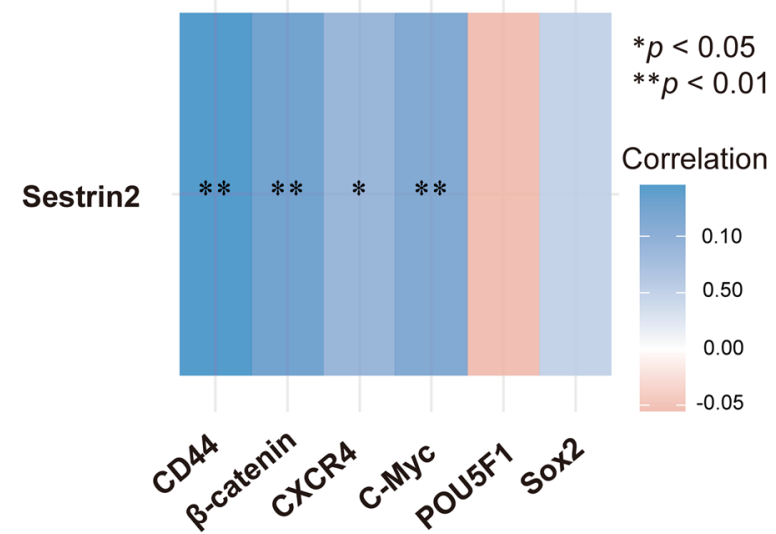

Fig. 4 (See legend on previous page.) 
molecules such as tanshinone IIA, fangchinoline, and nelfinavir work together with Sestrin2 to carry out these functions [42-44]. This study showed that Sestrin2 also has anticancer effects.

The effects of Sestrin2 on the Wnt pathway may occur via the AKT signaling pathway. Usually, Sestrin2, known as a p53 target gene, inhibits mTORC1 signaling and the induction of autophagy [45, 46]. Interestingly, our results indicated that Sestrin 2 also downregulates the Wnt/ $\beta$-catenin signaling pathway. The reasons why our results differ from others may be that SENS2 activates AKT and AMPK signaling [39] and PI3K/Akt/ Wnt/ $\beta$-catenin signaling, which controls the levels of EMT-related proteins [37]. Thus, Sestrin2 may affect $\beta$-catenin by interacting with upstream factors.

In conclusion, the results of this study showed that LV-Sesrin2 inhibits cancer stemness through the Wnt pathway in HCT-116 and SW620 cells, suggesting that Sesrin2 may be a promising therapeutic target for CRC.

\section{Supplementary Information}

The online version contains supplementary material available at https://doi. org/10.1186/s12935-022-02498-x.

Additional file 1: Figure S1. Cell invasion was detected by Transwell assay. The cell number was counted by ImageJ and normalized to the LV-GFP group (right panel) ${ }^{*} P=0.05$; Mann-Whitney test; lines showed medians).

Additional file 2: Figure S2. (A) The relative protein expression of Sestrin2, Sox2, Oct4, CxCr4, and CD44 in the LV-GFP and LV-Sestrin2 groups of HCT-116 and SW620 cells ( ${ }^{*} P=0.05$; Mann-Whitney test; lines showed medians). (B) Representative western blot images of the effect of LV-Sestrin2 on the expression levels of Sestrin2, Sox2, Oct4, and Cxcr4 in HCT-116 and SW620 sphere cells. $\beta$-actin was used as a loading control. The relative protein expression is on the right $\left({ }^{*} P=0.05\right.$; Mann-Whitney test; lines showed medians). (C) western blot images of the effect of LV-Sestrin2 on the expression levels of $\mathrm{E}$-cadherin. The relative protein expression is on the right ( ${ }^{*} P=0.05$; Mann-Whitney test; lines showed medians).

Additional file 3: Figure S3. Western blot images of the effect of LV-Sestrin2 with or without $48 \mathrm{~h}$ of treatment with scavengers of ROS, sodium pyruvate and carboxy-PTIO, on the expression levels of CD44 in HCT-116 and SW620 cells. $\beta$-actin was used as a loading control.

Additional file 4: Figure S4. (A) The mouse body weights were measured. (B) The relative protein expression of Sestrin2, CD44, Sox2, C-Myc, and $\beta$-catenin in mouse tumors $\left({ }^{*} P=0.05\right.$; Mann-Whitney test; lines showed medians).

Additional file 5: Table S1. The qPCR primer sequences used in the current study.

\section{Acknowledgements}

All authors thank Professor Weiyin Zhou from the College of Pharmacy, Chongqing Medical University for research equipment support.

\section{Authors' contributions}

JW and ZF designed the experiments. ZF supervised the whole project. XZ performed the major research and wrote the paper. $W L$ and $X L$ provide their professional guidance. XZ and WL provided the technical support. All authors read and approved the final manuscript.

\section{Funding}

This study was funded by the Chongqing Science and Technology Commission (No. cstc2017jcyjAX0127).

\section{Availability of data and materials}

The data and materials can be obtained from the first author and corresponding author.

\section{Declarations}

\section{Ethics approval and consent to participate}

This study was approved by the Ethics Committee of the First Affiliated Hospital of Chongqing Medical University.

\section{Consent for publication}

All authors consent to publication.

\section{Competing interests}

The authors declare that no competing interests exist.

\section{Author details}

${ }^{1}$ Department of Gastrointestinal Surgery, The First Affiliated Hospital of Chongqing Medical University, Chongqing 400016, China. ${ }^{2}$ The Third Affiliated Hospital of Chongqing Medical University, Chongqing, China. ${ }^{3}$ College of Pharmacy, Chongqing Medical University, Chongqing, China.

Received: 7 July 2021 Accepted: 30 January 2022

Published online: 11 February 2022

\section{References}

1. Chen W, Zheng R, Baade PD, et al. Cancer statistics in China, 2015. Cancer J Clin. 2016;66(2):115-32.

2. van der Heijden M, Vermeulen L. Stem cells in homeostasis and cancer of the gut. Mol Cancer. 2019;18(1):66.

3. Plaks V, Kong N, Werb Z. The cancer stem cell niche: how essential is the niche in regulating stemness of tumor cells. Cell Stem Cell. 2015;16(3):225-38.

4. Dehghani $\mathrm{H}$. Regulation of chromatin organization in cell stemness: the emerging role of long non-coding RNAs. Stem Cell Rev Rep. 2021;17(6):2042-53

5. Park HW, Park H, Ro SH, et al. Hepatoprotective role of Sestrin2 against chronic ER stress. Nat Commun. 2014;5:4233.

6. Budanov AV. Stress-responsive sestrins link p53 with redox regulation and mammalian target of rapamycin signaling. Antioxid Redox Signal. 2011;15:1679-90. https://doi.org/10.1089/ars.2010.3530.

7. Lee JH, Budanov AV, Karin M. Sestrins orchestrate cellular metabolism to attenuate aging. Cell Metab. 2013;18:792-801. https://doi.org/10.1016/j. cmet.2013.08.018.

8. Pasha M, Eid AH, Eid AA, Gorin Y, Munusamy S. Sestrin2 as a novel biomarker and therapeutic target for various diseases. Oxid Med Cell Longev. 2017;2017:3296294. https://doi.org/10.1155/2017/3296294.

9. Chae HS, Gil M, Saha SK, Kwak HJ, Park HW, Vellingiri B, et al. Sestrin2 expression has regulatory properties and prognostic value in lung cancer. J Pers Med. 2020. https://doi.org/10.3390/jpm10030109.

10. Shin J, Bae J, Park S, Kang HG, Shin SM, Won G, et al. mTOR-dependent role of sestrin 2 in regulating tumor progression of human endometrial cancer. Cancers. 2020. https://doi.org/10.3390/cancers12092515.

11. Wei JL, Fu ZX, Fang M, et al. Decreased expression of sestrin 2 predicts unfavorable outcome in colorectal cancer. Oncol Rep. 2015;33(3):1349-57.

12. Kim GT, Lee SH, Kim JI, Kim YM. Quercetin regulates the sestrin 2-AMPKp38 MAPK signaling pathway and induces apoptosis by increasing the generation of intracellular ROS in a p53-independent manner. Int J Mol Med. 2014;33(4):863-9.

13. Seo K, Seo S, Ki SH, Shin SM. Sestrin2 inhibits hypoxia-inducible factor-1a accumulation via AMPK-mediated prolyl hydroxylase regulation. Free Radic Biol Med. 2016;101:511-23. 
14. Agro L, O'Brien C. In vitro and in vivo limiting dilution assay for colorectal cancer. Bio Protoc. 2015;5:1-11.

15. Hu Y, Smyth GK. ELDA: extreme limiting dilution analysis for comparing depleted and enriched populations in stem cell and other assays. J Immunol Methods. 2009;347:70-8.

16. Liu Y, Zhang $X$, Han C, Wan G, Huang X, Ivan C, et al. TP53 loss creates therapeutic vulnerability in colorectal cancer. Nature. 2015;520:697-701.

17. Chandrashekar DS, Bashel B, Balasubramanya S, et al. UALCAN: a portal for facilitating tumor subgroup gene expression and survival analyses. Neoplasia. 2017;19(8):649-58.

18. Chang JC. Cancer stem cells: role in tumor growth, recurrence, metastasis, and treatment resistance. Medicine. 2016;95(1 Suppl 1):S20-5.

19. Li Y, Liu YH, Hu YY, Chen L, Li JM. Special AT-rich sequence-binding protein 2 acts as a negative regulator of stemness in colorectal cancer cells. World J Gastroenterol. 2016;22:8528-39. https://doi.org/10.3748/wjg.v22. i38.8528.

20. Cao L, Zhou Y, Zhai B, Liao J, Xu W, Zhang R, et al. Sphere-forming cell subpopulations with cancer stem cell properties in human hepatoma cell lines. BMC Gastroenterol. 2011;11:71.

21. Zare K, Shademan M, Ghahramani Seno MM, Dehghani H. CRISPR/Cas9 knockout strategies to ablate CCAT1 IncRNA gene in cancer cells. Biol Proced Online. 2018;20:21.

22. Horibata S, Vo TV, Subramanian V, Thompson PR, Coonrod SA. Utilization of the soft agar colony formation assay to identify inhibitors of tumorigenicity in breast cancer cells. J Vis Exp. 2015. https://doi.org/10.3791/ 52727.

23. Karsten U, Goletz $\mathrm{S}$. What makes cancer stem cell markers different. Springerplus. 2013;2(1):301.

24. Tomao F, Papa A, Rossi L, et al. Emerging role of cancer stem cells in the biology and treatment of ovarian cancer: basic knowledge and therapeutic possibilities for an innovative approach. J Exp Clin Cancer Res. 2013;32:48.

25. Deshmukh A, Deshpande K, Arfuso F, Newsholme P, Dharmarajan A Cancer stem cell metabolism: a potential target for cancer therapy. Mol Cancer. 2016:15(1):69.

26. Vlashi E, Pajonk F. Cancer stem cells, cancer cell plasticity and radiation therapy. Semin Cancer Biol. 2015;31:28-35.

27. Zhan T, Rindtorff N, Boutros M. Wnt signaling in cancer. Oncogene. 2017;36(11):1461-73.

28. Espada J, Calvo MB, Díaz-Prado S, Medina V. Wnt signalling and cancer stem cells. Clin Transl Oncol. 2009;11(7):411-27.

29. Koveitypour Z, Panahi F, Vakilian M, et al. Signaling pathways involved in colorectal cancer progression. Cell Biosci. 2019;9:97.

30. Najafi M, Farhood B, Mortezaee K. Cancer stem cells (CSCs) in cancer progression and therapy. J Cell Physiol. 2019;234(6):8381-95.

31. Khan AQ, Rashid K, AIAmodi AA, Agha MV, Akhtar S, Hakeem I, et al. Reactive oxygen species (ROS) in cancer pathogenesis and therapy: an update on the role of ROS in anticancer action of benzophenanthridine alkaloids. Biomed Pharmacother. 2021;143:112142.

32. Zhu G, Xu P, Guo S, Yi X, Wang H, Yang Y, et al. Metastatic melanoma cells rely on sestrin2 to acquire anoikis resistance via detoxifying intracellular ros. J Invest Dermatol. 2020;140:666-75.e2.

33. Jariyal H, Gupta C, Bhat VS, Wagh JR, Srivastava A. Advancements in cancer stem cell isolation and characterization. Stem Cell Rev Rep. 2019;15(6):755-73.

34. Eun K, Ham SW, Kim H. Cancer stem cell heterogeneity: origin and new perspectives on CSC targeting. BMB Rep. 2017;50(3):117-25.

35. Liu X, Fan D. The epithelial-mesenchymal transition and cancer stem cells: functional and mechanistic links. Curr Pharm Des. 2015;21(10):1279-91.

36. Jia $Y$, Zheng $Z$, Yang $Y$, et al. MiR-4756 promotes albumin-induced renal tubular epithelial cell epithelial-to-mesenchymal transition and endoplasmic reticulum stress via targeting sestrin2. J Cell Physiol. 2019;234(3):2905-15.

37. Lee $\mathrm{K}$, Chang JW, Oh $\mathrm{C}$, et al. HOXB5 acts as an oncogenic driver in head and neck squamous cell carcinoma via EGFR/Akt/Wnt/ $\beta$-catenin signaling axis. Eur J Surg Oncol. 2019;46(6):1066-73.

38. Ro SH, Xue X, Ramakrishnan SK, et al. Tumor suppressive role of sestrin2 during colitis and colon carcinogenesis. Elife. 2016;5:e12204.

39. Dai J, Huang $Q$, et al. Sestrin 2 confers primary resistance to sorafenib by simultaneously activating AKT and AMPK in hepatocellular carcinoma. Cancer Med. 2018;7(11):5691-703.
40. Xu H, Sun $H$, Zhang H, et al. An ShRNA based genetic screen identified sesn2 as a potential tumor suppressor in lung cancer via suppression of Akt-mTOR-p70S6K signaling. PLoS ONE. 2015;10(5):e0124033.

41. Zhao B, Shah P, Budanov AV, et al. Sestrin2 protein positively regulates AKT enzyme signaling and survival in human squamous cell carcinoma and melanoma cells. J Biol Chem. 2014;289(52):35806-14.

42. Bruning A, Rahmeh M, Friese K. Nelfinavir and bortezomib inhibit mTOR activity via ATF4-mediated sestrin-2 regulation. Mol Oncol. 2013;7(6):1012-8.

43. Wang N, Pan W, Zhu M, et al. Fangchinoline induces autophagic cell death via p53/sestrin2/AMPK signalling in human hepatocellular carcinoma cells. Br J Pharmacol. 2011;164(2b):731-42.

44. Yen JH, Huang ST, Huang HS, et al. HGK-sestrin 2 signaling-mediated autophagy contributes to antitumor efficacy of Tanshinone IIA in human osteosarcoma cells. Cell Death Dis. 2018;9(10):1003.

45. Liang Y, Zhu J, Huang H, et al. SESN2/sestrin 2 induction-mediated autophagy and inhibitory effect of isorhapontigenin (ISO) on human bladder cancers. Autophagy. 2016;12(8):1229-39.

46. Wolfson RL, Chantranupong L, Saxton RA, et al. Sestrin2 is a leucine sensor for the mTORC1 pathway. Science. 2016;351(6268):43-8.

\section{Publisher's Note}

Springer Nature remains neutral with regard to jurisdictional claims in published maps and institutional affiliations.

Ready to submit your research? Choose BMC and benefit from:

- fast, convenient online submission

- thorough peer review by experienced researchers in your field

- rapid publication on acceptance

- support for research data, including large and complex data types

- gold Open Access which fosters wider collaboration and increased citations

- maximum visibility for your research: over $100 \mathrm{M}$ website views per year

At BMC, research is always in progress.

Learn more biomedcentral.com/submissions 